\title{
A case of secondary syphilis mimicking palmoplantar psoriasis in HIV infected patient*
}

\author{
Maraya de Jesus Semblano Bittencourt ${ }^{1}$ \\ Bianca Angelina Macêdo do Nascimento ${ }^{1}$ \\ Manoel Dias do Nascimento ${ }^{1}$
}

\author{
Arival Cardoso de Brito ${ }^{1}$ \\ Alessandra Haber Carvalho ${ }^{1}$
}

DOI: http:/ / dx.doi.org/10.1590/abd1806-4841.20153399

\begin{abstract}
A bstract: Due to diverse clinical and histopathological presentations, diagnosis of secondary syphilis can occasionally prove challenging. Variable clinical presentations of secondary syphilis in HIV disease may result in an incorrect diagnosis and an inappropriate treatment regimen. Similarly, the histology of secondary syphilitic lesions may show considerable variation, depending on the clinical morphology of the eruption. We report a case of secondary syphilis in an HIV infected patient with cutaneous palmoplantar lesions simulating palmoplantar psoriasis.
\end{abstract}

Keywords: Histology; Nails; Psoriasis; Syphilis; Treponemal infections; Treponema pallidum

\section{INTRODUCTION}

Syphilis is a sexually transmitted disease caused by the Treponema pallidum, whose prevalence has been increasing worldwide. It is considered "the great imitator" due to its varied presentations. The HIV virus has many effects on clinical presentation, diagnosis, disease progression and treatment of syphilis. ${ }^{1}$ Even though syphilis presentation in these patients is for the most part similar to that of patients without HIV, atypical manifestations of the disease may be present, including severe and rare forms. ${ }^{2-5}$

\section{CASE REPORT}

Male patient, 32 years old, has been presenting bilateral asymptomatic erythematous-scaly plaques on palms and soles for three months, associated with periungual erythema, subungual hyperkeratosis and onychodystrophy of toenails (Figures 1 and 2). He had no previous history of skin disease or other comorbidities and was not using any medication. Direct mycological examination of cutaneous lesions and of all nails involved resulted negative. Biopsy of left palm lesion showed epidermis with

1 Universidade Federal do Pará (UFPA) - Belém (PA), Brazil. 


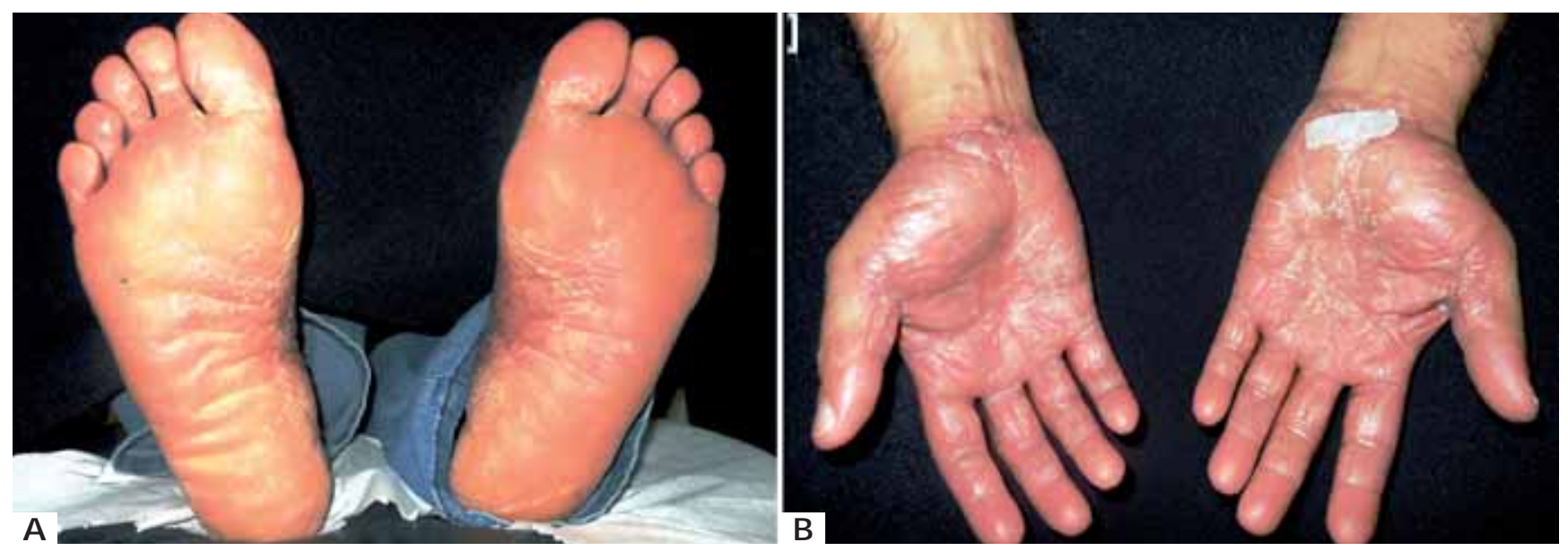

FiguRE 1: A. Erythematous-scaly plaque on soles. B. Erythematous-scaly plaque on palms
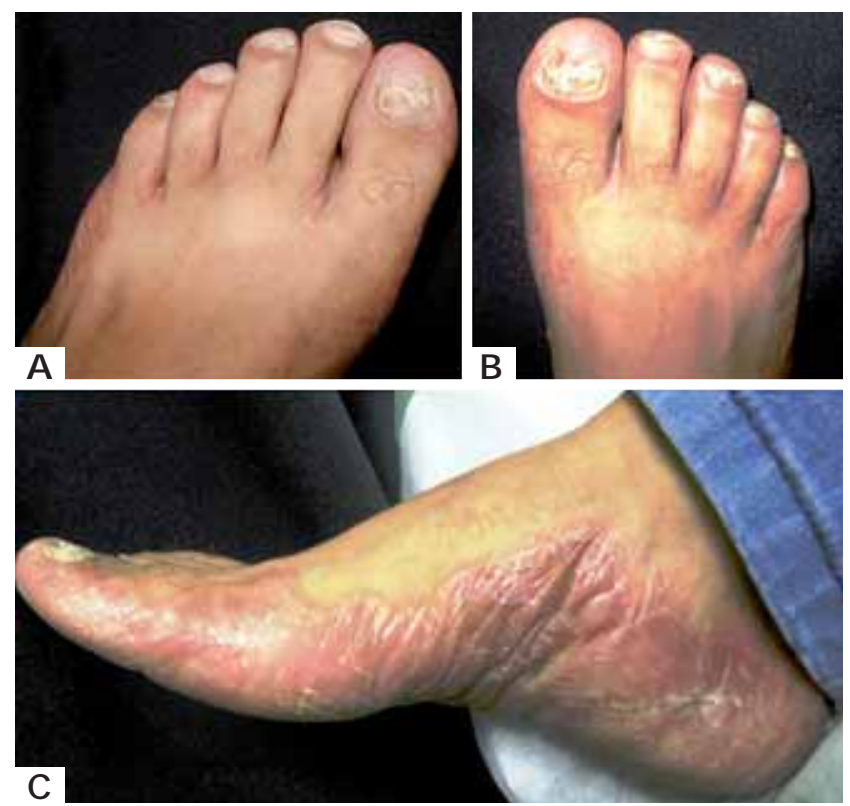

Figure 2: A. Periungual erythema and edema, subungueal hyperkeratosis and onychodystrophy of toenails.

B. infiltrated erythematous-scaly plate, circinated, on the sole and dorsal part of right foot
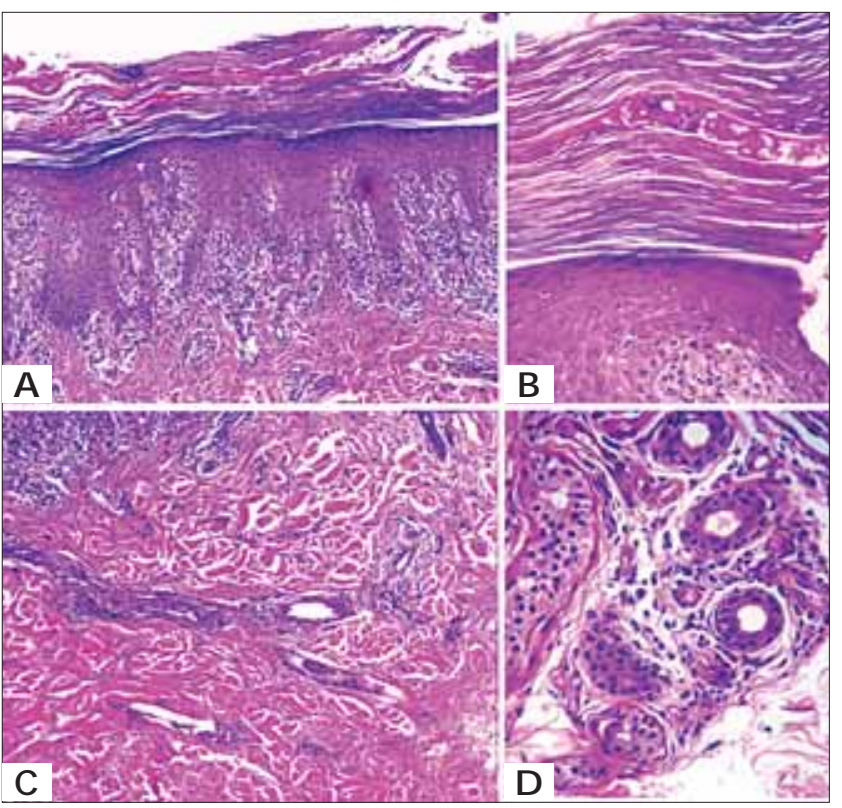

Figure 3: A. Psoriasiform hyperplasia and lichenoid infiltrate which darkens the dermal-epidermal interface, a mix of lymphocytes, histiocytes, plasmocytes and neutrophils (H\&E, x10). B. Compact hyperkeratosis, parakeratosis and intracorneal neutrophils (H\&E, x10). C. Lymphohistiocytic interstitial infiltrate, superficial and deep (H\&E, x40). D. Inflammatory infiltrate involving deep annexes (H\&E, x40)

superficial and deep interface dermatitis suggested the inclusion of treponematosis among the diagnostic possibilities. The patient returned with the following laboratory results: VDRL 1/256 and anti HIV 1 and 2 antibodies: 1003.37, confirming the diagnosis of secondary syphilis and HIV coinfection. CD4 levels were 185 and viral load 800,000 copies/ml. Treatment with benzathine penicillin 2,400,000 U per week was prescribed for three weeks, with complete remission 

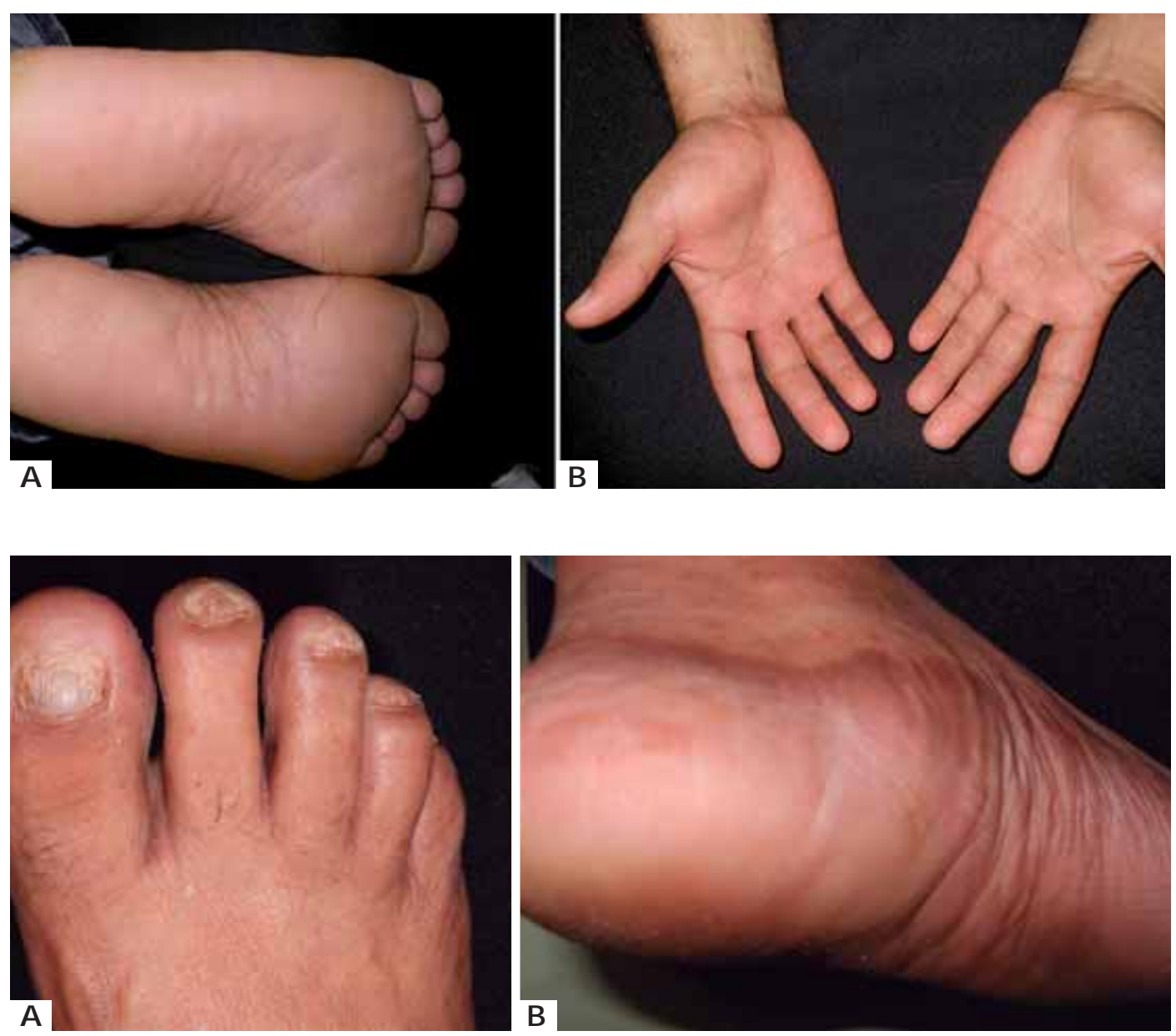

Figure 4:

A and B. Clinical aspect of palmoplantar lesions 1 week after completion of treatment with benzathine penicillin

Figure 5:

A and B. Clinical aspect of ungual lesions 1 week after completion of treatment with benzathine penicillin of lesions; the patient was sent to a REFERENCES center for HIV virus treatment (Figures 4 and 5). New nail mycological examination was performed upon his return and the result was negative.

\section{DISCUSSION}

According to World Health Organization data, there are approximately 900,000 new cases of syphilis detected annually in Brazil, and this fact reiterates the need for vigilance. Interactions between syphilis and HIV virus arise from the fact that both are transmitted mainly through sexual relations and their importance is increased because ulcerated genital lesions augment the risk of contracting and transmitting the HIV virus; for this reason all patients with syphilis should be offered HIV tests and all HIV-positive patients must be regularly tested for syphilis., ${ }^{2,6}$

In HIV-infected patients syphilis does not present opportunistic behavior, but has less usual clinical characteristics, more aggressive and exuberant, with more frequent and early involvement of the nervous system. ${ }^{3,4,5,7}$ Cutaneous lesions are the most common clinical manifestation of secondary syphilis $(72 \%)$, and may be the only manifestation of the disease. ${ }^{7}$

Cutaneous manifestations are so variable that syphilis must be considered in the diagnosis of all dermatoses with atypical presentation. The involvement of palmar and plantar regions is very characteristic and erythematous papules with a scaly collarette are the most common presentation. Sometimes scaling is intense, giving a psoriasiform aspect to lesions. ${ }^{6}$ Ungual involvement is rarely described in literature and includes nail plate and periungual tissue alterations. ${ }^{8}$ The negative result of direct mycological examination of nails, the absence of ungual alterations before the onset of cutaneous lesions and excellent improvement of nail plate and periungual tissue by the end of treatment suggested to the authors that these alterations were associated with syphilis.

Due to the several clinical presentations, a biopsy is usually performed and the diagnosis established, as in our case. Histological characteristics of secondary syphilis are very variable and show epidermal and/or dermal changes. In general, the inflammatory reaction acquires a lichenoid (lichen planus type) and/or psoriasiform (psoriasis type) pattern. Findings with 
lichenoid psoriasiform aspect, as observed in our case, present a dense mononuclear infiltrate which extends itself to the deep dermis. ${ }^{9}$ The infiltrate is composed of lymphocytes, macrophages, plasmocytes, neutrophils and eosinophils in variable proportions. Associated vascular alterations include vascular proliferation, prominence of endothelial cells under light, associated with perivascular infiltrate. Specific Wharthin-Starry staining (silver staining) can identify the treponema in the tissue. ${ }^{4}$

Serological tests in the majority of HIV-infected patients are within the patterns found in noninfected patients. ${ }^{6}$ However, atypical results may occur. Titration may be too high or too low, and false negativity may occur in some cases. ${ }^{6}$ Several studies suggest that syphilis, like many other acute infections, leads to transient viral load increases and diminished CD4 cell count, which are resolved after the infection

\section{REFERENCES}

1. Golden MR, Marra CM, Holmes KK. Update on syphilis: resurgence of an old problem. JAMA. 2003;290:1510-4.

2. Zetola NM, Klausner JD.Syphilis and HIV infection: an update. Clin Infect Dis. 2007:44:1222-8

3. Lynn WA, Lightman S. Syphilis and HIV: a dangerous combination. Lancet Infect Dis. 2004:4:456-66.

4. Liotta EA, Turiansky GW, Berberian BJ, Sulica VI, Tomaszewski MM. Unusual presentation of secondary syphilis in 2 HIV-1 positive patients. Cutis. 2000;66:383-6, 389.

5. Gutierrez-Galhardo MC, do Valle GF, Sá FC, Schubach Ade 0, do Valle AC. Clinical characteristics and evolution of syphilis in 24 HIV + individuals in Rio de Janeiro, Brazil. Rev Inst Med Trop Sao Paulo. 2005;47:153-7.

6. Avelleira JC, Bottino G. Syphilis: diagnosis, treatment and control. An Bras Dermatol. 2006:81:111-26.

7. Kumar B, Gupta S, Muralidhar S. Mucocutaneous manifestations of secondary syphilis in north Indian patients: a changing scenario? J Dermatol. 2001;28:13744.

8. Oh IY, Kim H, Park KY, Li K, Kim BJ, Seo SJ, et al. A Case of Syphilid with Nail Dystrophy. Korean J Dermatol. 2012;50:628-31.

9. Engelkens HJ, ten Kate FJ, Vuzevski VD, van der Sluis JJ, Stolz E. Primary and secondary syphilis: a histopathological study. Int J STD AIDS. 1991;2:280-4.

10. Kofoed K, Gerstoft J, Mathiesen LR, Benfield T. Syphilis and human immunodeficiency virus (HIV)-1 coinfection: influence on CD4 T-cell count, HIV-1 viral load, and treatment response. Sex Transm Dis. 2006;33:143-8. is treated. ${ }^{10}$ It is possible that these transient viral load increases contribute to magnification of HIV transmission risk among patients with syphilis and HIV. ${ }^{10}$ The treatment in the case presented was performed with intramuscular benzathine penicillin $7,200,000 \mathrm{UI}$, in three weekly doses of 2,400,000UI, since it was a case of syphilis of undetermined duration. ${ }^{2}$

Syphilis is still a serious public health problem and we must be alert to this diagnosis, especially in case of atypical cutaneous clinical pictures in which they simulate other cutaneous diseases. We present a case of secondary syphilis which clinically simulated psoriasis and dermatophytosis, showing ungual alterations that suggest syphilis involvement, due to the negative result of mycological examination and improvement after treatment. The association with the HIV virus must always be investigated, as in the case presented, due to its association with atypical manifestations of secondary syphilis.

\author{
M AILING ADDRESS: \\ $M$ araya de Jesus Semblano Bittencourt \\ Travessa Vileta 1289, A pt. 301 \\ Pedreira \\ 66087-422 - Belém - PA \\ Brazil \\ E-mail: marayabittencourt@hotmail.com
}

H ow to cite this article: Bittencourt MJS, Brito AC, Nascimento BAM, Carvalho AH, Nascimento MD. A case of secondary syphilis mimicking palmoplantar psoriasis in HIV infected patient. An Bras Dermatol. 2015;90 (3 Suppl 1):S216-9. 